\title{
Laboratory simulations of coastally trapped waves with rotation, topography and stratification
}

\author{
Peter G. Baines ${ }^{\mathrm{a}, *}$, Don L. Boyer ${ }^{\mathrm{b}}$, Bin Xie $^{\mathrm{b}}$ \\ a Department of Civil and Environmental Engineering, University of Melbourne, Victoria 3010, Australia \\ b Environmental Fluid Dynamics Program, Department of Mechanical and Aerospace Engineering, \\ Arizona State University, Tempe, AZ, USA
}

Received 25 February 2004; received in revised form 12 December 2004; accepted 17 December 2004

\begin{abstract}
We describe observations of the generation and propagation of coastally trapped waves in the laboratory and their comparison with theory, over a range of values of several experimental parameters. The topography and stratification used consisted of a sloping continental shelf and vertical continental slope with three-layer stratification that could be approximated by an extended version of the Gill and Clarke model [Gill, A.E., Clarke, A., 1974. Wind-induced upwelling, coastal currents and sea level changes. Deep Sea Res. 21, 325-345]. The latter was modified to accommodate a central mixed layer, curved geometry, and friction on the shelf. This configuration represents coastal geometry with large Burger number. The experiments were successful in realizing coastally trapped waves that were consistent with the theoretical expectations. However, the waves propagated more slowly, and for narrow shelves were damped more rapidly than predicted by the theory. The first was attributed to: (i) the effect of stratification on fluid on the shelf, reducing the topographic Rossby wave effect; (ii) the parameterization of the viscosity. The second difference was attributed to the mechanism of generation: the paddle used did not always generate sinusoidal waves, and the subsequent dispersion resulted in a net loss of amplitude.
\end{abstract}

(c) 2005 Elsevier B.V. All rights reserved.

Keywords: Topography; Amplitude; Coastal

\footnotetext{
* Corresponding author.

E-mail address: p.baines@ civenv.unimelb.edu.au (P.G. Baines).
} 


\section{Introduction}

It is well established that in coastal waters, wind and tidal forcing at subinertial frequencies can generate waves that propagate along the continental slopes and shelves of the world's coastlines as coastal-trapped waves (CTWs). Among the earliest observations of CTWs were those of Hamon $(1962,1963,1966)$ in which he analyzed data taken during June 1957 to December 1958 along both the eastern and western Australian coasts. Hamon's interpretation of the data as being of the nature of CTWs was supported by an analysis by Robinson (1964). The phase speed of these waves is in a direction with the coast on the right (left), facing downstream, in the northern (southern) hemisphere.

CTWs generated by alongshore wind stresses due to storms, for example, have typical periods ranging from days to weeks. Reviews by Allen (1980) and Brink (1991, 1998a) have described the observational evidence for the existence of CTWs, and the linear theory that describes their generation and propagation. The ubiquity of these waves is demonstrated by observations of them in the following regions: the eastern coast of Australia (Freeland et al., 1986; Church and Freeland, 1987), the east coast of North America (Noble and Butman, 1979; Yankovsky and Garvine, 1998), the Oregon and Washington coasts (Battisti and Hickey, 1984; Chapman, 1987), the Peru coast (Smith, 1978; Enfield et al., 1987; Clarke and Ahmed, 1999) and the West Florida shelf (Mitchum and Clarke, 1986). Poleward of $30^{\circ}$ latitude, they may also be generated by the interaction of diurnal tides with topographic features such as the Strait of Juan de Fuca (Crawford and Thomson, 1984; Flather, 1988) and the Gulf of Maine (Daifuku and Beardsley, 1983). CTWs may also arise from the equatorial wave guide where this intersects a west coast coastline, producing waves with long periods ranging from several days to months and even years (Brink, 1998b).

The linear theory for the propagation of CTWs for the case of a stably stratified background fluid and a continental shelf-slope topographic profile independent of the alongshore coordinate is well developed (e.g., Huthnance, 1978; Brink, 1991). The dependent variables to be determined are the velocity components $(u, v, w)$ and the perturbation pressure and density functions ( $p$ and $\rho$ ); the independent variables are the rectangular Cartesian coordinates $(x, y, z)$ in the off-shore, along-shore (with the coast on the left) and vertically upward directions, respectively, and the time $t$. Solutions for free CTWs are obtained by assuming solutions of the form $p=\exp [\mathrm{i}(\omega t+k y)] F(x, z)$, where $\omega$ is the frequency of the disturbance and $k$ the along-shore wavenumber. Thus, for a given wavenumber $k$, the problem is reduced to an eigenvalue problem for $F(x, z)$ and $\omega$. The modal structures can be found via resonance iteration (Wang and Mooers, 1976; Brink, 1982; Brink and Chapman, 1987), or inverse iteration (Huthnance, 1978).

CTWs may take a variety of different forms. For example, the dimensionless governing equations lead to the introduction of the Burger number defined as $B u=(N H / f L)^{2}$, where $H$ is the fluid depth, $N$ the buoyancy frequency, $f$ the Coriolis parameter and $L$ the cross-shelf characteristic length scale. The Burger number can be interpreted as the ratio of the internal radius of deformation to the horizontal length scale, and its value characterizes the type of CTWs that may occur. When $B u$ is small, the waves resemble barotropic topographic Rossby waves (known as continental shelf waves), but when $\mathrm{Bu}$ is large they more resemble internal Kelvin waves (Huthnance, 1978). When $k L$ is large, they resemble bottom edge waves of the form described by Rhines (1970). 
Brink (1991), obtained the dispersion relations for the free-wave mode solutions. For arbitrary topography (but with depth increasing away from the coast) and stratification, the wave with the highest frequency, or the greatest speed, is similar to a barotropic Kelvin wave. This may be termed mode 0 , and is not of concern here. The CTWs of interest comprise an ordered infinite set of additional modes, each with successively slower speed and more complex structure. When stratification can be neglected, all these free-wave modes exist for all alongshore wave numbers and their frequencies are always less than the inertial frequency; these are commonly known as shelf waves. As the stratification is increased, the wave frequency for each mode at a given wave number increases until it reaches the inertial frequency. When the wave frequency goes beyond $f$, the wave is no longer trapped against the coastline and may propagate into deeper water. In this linear CTW framework, each mode propagates independently of the others, and is forced by the projection of the (wind) forcing on it. If the forcing and the resulting waves have a large enough length scale, the waves are non-dispersive and are governed by the so-called "first-order wave equation" (FOWE); see, for example, Clarke and Van Gorder (1986). Under this approximation, wave periods are in the range of several days to a few weeks and along-shelf motion scales are much longer than the shelf width. FOWE studies have been applied in studies by Battisti and Hickey (1984), Church et al. (1986) and Chapman (1987) among others.

Most coastlines contain along-shelf spatial variations that are much shorter than CTW along-shelf length scales. Not a great deal is known about the effects of such variations on the CTWs. Wilkin and Chapman $(1987,1990)$ conducted analytical studies of the scattering of CTWs incident on a region where the coastal wave guide becomes suddenly wider or narrower, in both barotropic and stratified model oceans. In general, an incident wave is scattered into a number of other CTW modes by such an encounter.

Other factors that bear on the usefulness of the linear framework are bottom friction, and nonlinearity. The laboratory experiments discussed below demonstrate that both nonlinearity and bottom friction may be important in certain regions of parameter space, and we expect this to be the case for the oceans. Large amplitude forcing by alongshore wind stress is probably common, but what is the effect of this on the generation of CTWs? One would expect nonlinearities to become important when forcing becomes sufficiently large, but there are no studies that suggest how large this is. What is the response when this limit is exceeded, and what is the expected form of large-amplitude CTWs? Bottom friction is better understood, and is known to be important on shallow shelves (Csanady, 1978). It may also be more effective at shorter wavelengths where the waves have larger amplitude close to the bottom, as in Rhines (1970), but this situation is complicated by the "slippery boundary" phenomenon (e.g., Garrett et al., 1993).

The subject of coastal-trapped waves is virtually unexplored in the laboratory. Whitehead and Chapman (1986), in an investigation of gravity currents, observed that when the current speed was less than that of the first-mode barotropic shelf-wave, a shelf-wave was radiated ahead of the gravity current. The only previous laboratory study of CTWs (with rotation, topography and stratification) known to us is that of Codiga et al. (1999), who reported detailed observations of one particular case, using shelf-slope topography with uniform stratification, using the $13 \mathrm{~m}$ diameter turntable of the Coriolis Laboratory in Grenoble, France. They found, in particular, that CTWs, whose Rossby radii were much larger than the canyon width, were very little affected by their passage across the canyon. 
Apart from field observations, most theoretical work on CTWs has been with linear models. Comparatively little effort has been directed toward the effects of large amplitude forcing on such waves, and the associated limitations of the CTW "paradigm". Bottom friction is also known to be important, and decay rates may be quite large if the continental shelf is wide and shallow (Csanady, 1978). Alongshore topographic variations may degrade or scatter the wave energy. Alongshore currents and current shear that vary on frequencies lower than the CTWs, if present at sufficient amplitude, may also affect the wave properties and cause propagation to cease altogether.

The experiments reported here were done to investigate some basic properties of coastally trapped waves, and to see how well they were described by simple dynamical models. These properties included possible finite amplitude effects, and bottom friction. A second objective was to explore the feasibility of using laboratory models to study CTWs and their interaction with coastal currents. How easy is it to generate them in a realistic laboratory simulation? This paper does not resolve all these issues, but is at least a start in this direction.

The present experiments employ a particular idealised topography, of a sloping shelf and vertical continental slope, with three-layer stratification containing homogeneous top and bottom layers. This system permits a limiting form of CTW that consists of an internal Kelvin wave in the deep water, coupled to a topographic Rossby wave over the shelf. It has the advantage that analytical models exist for these CTWs, and bottom friction on the shelf may be readily included in them. In the present experiments we cover a wide range of parameter values, covering some 100+ runs, which give an initial indication of the properties of CTWs in the laboratory, and the factors that affect them. We first describe the theoretical model that has been developed for the purpose of providing a dynamical description of the CTWs as produced in the tank, in Section 2. We then proceed to describe the experimental arrangement used (Section 3), and the properties of the CTWs that were produced (Section 4). Comparisons are made with the theory, and the conclusions are described in Section 5. A preliminary description of some of these results was presented in Baines et al. (2001), hereafter designated BBXM.

\section{Theoretical model}

The model employed here is the two-layer model of Gill and Clarke (1974) adapted to circular geometry, three layers and the inclusion of a longshore current and friction on the continental shelf. To conform with the experiments, a polar coordinate system $(r, \theta)$ was used with the origin at the center of the tank, and the coast at the outside tank wall, radius $r=r_{\mathrm{b}}$; the continental slope is at $r=r_{\mathrm{a}}$. Consistent with the Gill and Clarke model for a rectilinear coastline, the topography for the experiment was constructed with the cross-sectional shelf depth of

$$
D(r)=D_{\mathrm{s}}-5\left(1-e^{-2 \lambda\left(r-r_{\mathrm{a}}\right)}\right), \quad r_{\mathrm{a}}<r<r_{\mathrm{b}},
$$

where all lengths and distances are in centimeters. However, it turns out that it is more convenient to approximate this expression with a power law of the form

$$
D(r)=D_{\mathrm{s}}\left(\frac{r_{\mathrm{a}}}{r}\right)^{\mu} .
$$


Equating the values of $D$ at the end points $r=r_{\mathrm{a}}, r_{\mathrm{b}}$ for (2.1) and (2.2) may be achieved by

$$
\mu=\frac{\ln \left(D_{\mathrm{s}} / D\left(r_{\mathrm{b}}\right)\right)}{\ln \left(r_{b} / r_{\mathrm{a}}\right)},
$$

which also gives reasonable agreement (within a few percent) for the values in between, for the parameter values of these experiments. At the rotation period of the experiments (20 s) the variation of depth over the continental shelf due to the sloping free surface is less than $1 \mathrm{~mm}$, and has been neglected.

In Gill and Clarke's model the fluid consisted of an upper layer of depth $D_{1}=D_{\mathrm{s}}$ and density $\rho_{1}$, and a lower layer of depth $D_{2}$, and density $\rho_{2}$, where the level of the interface coincided with the depth of the shelf at the shelf-break. In the experiments to be described below, the stratification was established by salt water and some mixing was unavoidable in the filling process. The resulting stratification usually consisted of an interfacial region of finite thickness. In order to take account of this stratified middle layer, the two-layer model was modified to include a uniformly stratified middle layer of thickness $D_{\mathrm{m}}$ with density that joined continuously with that of the homogeneous layers above and below. The density profile in the deep water is therefore

$$
\rho(z)= \begin{cases}\rho_{1} & -D_{1}<z<0, \\ \frac{\rho_{1}-\Delta \rho\left(z+D_{1}\right)}{D_{\mathrm{m}}}, & -\left(D_{1}+D_{\mathrm{m}}\right)<z<-D_{1}, \\ \rho_{2} \equiv \rho_{1}+\Delta \rho, & -D_{2}<z<-\left(D_{1}+D_{\mathrm{m}}\right) .\end{cases}
$$

The total depth is then given by $D=D_{1}+D_{\mathrm{m}}+D_{2}$, and the depth of the shelf break is $D_{\mathrm{s}}=D\left(r_{\mathrm{a}}+\right)$. In the experiments, $D_{1}$ is generally smaller than $D_{\mathrm{s}}$, but in equations for the motion on the shelf, the fluid there is assumed to be homogeneous, with the density of the upper layer.

For the undisturbed state, we assume that the fluid in the deep water is at rest, but the fluid over the shelf may be in longshore motion with velocity:

$$
U_{\theta}=r \Omega_{0},
$$

which is in rigid rotation with angular velocity $\Omega_{0}$, which may be positive or negative. This can represent a wind-driven current on the continental shelf. We model the bottom stress with a linear drag law (Brink, 1990) of the form

$$
\boldsymbol{\tau}=\rho R_{\mathrm{d}} \mathbf{u},
$$

where $R_{\mathrm{d}}$ is a constant. The value of $R_{\mathrm{d}}$ may be estimated from the conventional expression for the bottom stress, in the form:

$$
\boldsymbol{\tau}=\rho \nu \frac{\partial \mathbf{u}}{\partial z} \approx \rho \nu \frac{\mathbf{u}}{\delta}=\rho(\nu f)^{1 / 2} \mathbf{u},
$$

where $\delta$ is the Ekman boundary layer thickness, $(v / f)^{1 / 2}$. Hence $R_{\mathrm{d}}=(v f)^{1 / 2}$, which for these experiments, with $\nu=0.01 \mathrm{~cm}^{2} \mathrm{~s}^{-1}, f=0.628 \mathrm{~s}^{-1}$, gives $R_{\mathrm{d}}=0.08 \mathrm{~cm} \mathrm{~s}^{-1}$. If the perturbation fluid velocities in the directions of $(r, \theta)$ increasing are $u_{r}, u_{\theta}$, respectively, the equations of 
motion over the shelf are

$$
\begin{aligned}
& \left(\frac{\partial}{\partial t}+\Omega_{0} \frac{\partial}{\partial \theta}+\frac{R_{\mathrm{d}}}{D}\right) u_{r}-\left(f+2 \Omega_{0}\right) u_{\theta}=-g \frac{\partial \eta}{\partial r}, \\
& \left(\frac{\partial}{\partial t}+\Omega_{0} \frac{\partial}{\partial \theta}+\frac{R_{\mathrm{d}}}{D}\right) u_{\theta}+\left(f+2 \Omega_{0}\right) u_{r}=-\frac{g}{r} \frac{\partial \eta}{\partial \theta},
\end{aligned}
$$

where $g$ denotes acceleration due to gravity and $\eta$ the (small) free surface perturbation, so that $g \eta$ represents the barotropic pressure field. Note that friction on the sidewall at the coast, and on the vertical continental slope, have been neglected here. This is because the coastal sidewall has a height of order $2 \mathrm{~cm}$, which is much less than the shelf width, and friction on the (vertical) continental slope only affects the deep lower layer, where the velocities are half those of the upper. Hence these affects are expected to be substantially smaller than that of friction over the continental shelf.

We divide the fluid motion into the sum of barotropic and baroclinic components, and first consider the barotropic motion in the tank. Since the vertical displacement of the free surface, $\eta$, is small, it may be neglected in the continuity equation for the barotropic motion (the rigid lid approximation). The barotropic velocity $\overline{\boldsymbol{u}}$ may be then be expressed in terms of a Stokes stream function $\Psi$, in the manner

$$
\bar{u}_{r}=-\frac{1}{r D} \frac{\partial \Psi}{\partial \theta}, \quad \bar{u}_{\theta}=\frac{1}{D} \frac{\partial \Psi}{\partial r},
$$

where $D=D(r)$ on the shelf, and $D=D_{1}+D_{\mathrm{m}}+D_{2}$ is constant in deep water. We consider motions that have specified frequency $\omega$ and alongshore angular wavenumber $m$, and hence have dependence on $\theta$ and $t$ of the form $\mathrm{e}^{\mathrm{i}(m \theta-\omega t)}$. The equations for barotropic motion over the shelf are given by (2.8) and (2.9), and in deep water by the same equations, omitting the $\Omega_{0}$ and $R_{\mathrm{d}}$ terms. On the shelf, writing

$$
R=\frac{r}{r_{\mathrm{b}}}, \quad X=\left(\frac{r}{r_{\mathrm{b}}}\right)^{\mu}, \quad S=\frac{(v f)^{1 / 2}}{\omega D_{\mathrm{s}}}\left(\frac{r_{\mathrm{b}}}{r_{\mathrm{a}}}\right)^{\mu}=\frac{(v f)^{1 / 2}}{\omega D\left(r_{\mathrm{b}}\right)},
$$

where $S$ is the friction parameter, the vorticity equation becomes

$$
\begin{aligned}
& (1+\mathrm{i} S X) \mu^{2} X^{2} \Psi_{X X}+\left[\mu^{2}+1+\mathrm{i} S X\left(\mu^{2}+\mu+1\right)\right] X \Psi_{X} \\
& \quad+\left(M^{2}+\mu^{2} / 4-\mathrm{i} m^{2} S X\right) \Psi=0,
\end{aligned}
$$

where

$$
M^{2}=\frac{\mu m\left(f+2 \Omega_{0}\right)}{\omega-m \Omega_{0}}-m^{2}-\frac{\mu^{2}}{4} .
$$

If $S<1$ this equation has the series solution $\Psi=\Psi_{\mathrm{s}}(r) \mathrm{e}^{\mathrm{i}(m \theta-\omega t)}$, where

$$
\Psi_{\mathrm{s}}(r)=R^{\alpha_{+}}\left(1+\sum_{n=1}^{\infty} b_{n}\left(\alpha_{+}\right) R^{n \mu}\right)-\Re(m) R^{\alpha_{-}}\left(1+\sum_{n=1}^{\infty} b_{n}\left(\alpha_{-}\right) R^{n \mu}\right),
$$


(the subscript "s" standing for "shelf"), and

$$
\begin{aligned}
& \alpha_{ \pm}=-\frac{\mu}{2} \pm \mathrm{i} M, \\
& b_{0}=1, \quad b_{n}(\alpha)=-\mathrm{i} S b_{n-1}(\alpha) \frac{(\alpha+(n-1) \mu)^{2}+2 \mu(\alpha+(n-1) \mu)-m^{2}}{(n \mu)^{2}+n \mu(2 \alpha+\mu)} \\
& n>1 .
\end{aligned}
$$

From the condition $\Psi=0$ at $R=1$ :

$$
\Re(m)=\frac{1+\sum_{n=1}^{\infty} b_{n}\left(\alpha_{+}\right)}{1+\sum_{n=1}^{\infty} b_{n}\left(\alpha_{-}\right)} .
$$

For the inviscid case where $S=0$, this becomes

$$
\Psi_{\mathrm{s}}=2 \mathrm{i} r^{-\mu / 2} \sin \left(M \ln \left(\frac{r}{r_{\mathrm{b}}}\right)\right) .
$$

Viscous drag deforms this structure, as given by (2.14). The vorticity equation for $\Psi$ then has solutions of the form

$$
\Psi= \begin{cases}A_{1} r^{m} \mathrm{e}^{\mathrm{i}(m \theta-\omega t)}, & 0<r<r_{\mathrm{a}}, \\ A_{2} \Psi_{\mathrm{s}}(r) \mathrm{e}^{\mathrm{i}(m \theta-\omega t)}, & r_{\mathrm{a}}<r<r_{\mathrm{b}},\end{cases}
$$

where $A_{1}$ and $A_{2}$ are related by continuity of $\Psi$. This is the total motion over the coastal shelf, but in the deep water we also have the baroclinic component.

In the deep water, the baroclinic motion is determined by the conventional hydrostatic equation for the vertical velocity, $w$ :

$$
\left(\frac{\partial^{2}}{\partial t^{2}}+f^{2}\right) \frac{\partial^{2} w}{\partial z^{2}}+N^{2} \nabla_{\mathrm{h}}^{2} w=0,
$$

where $\nabla_{\mathrm{h}}$ is the horizontal Laplacian. The solution for $w$ then has the form

$$
w=A_{\mathrm{h}} \hat{w}(z) I_{m}(K r) \mathrm{e}^{\mathrm{i}(m \theta-\omega t)},
$$

where $I_{m}$ is the Bessel function of imaginary argument (Abramowitz and Stegun, 1965):

$$
K=\left(\frac{f^{2}-\omega^{2}}{g^{\prime} / D_{m}}\right)^{1 / 2} l_{\mathrm{h}},
$$

$g^{\prime}=g \Delta \rho / \rho_{1}$, and $l_{\mathrm{h}}$ depends only on the layer thicknesses and is given by

$$
\left(l_{\mathrm{h}}^{2} D_{1} D_{2}-1\right) \tan l_{\mathrm{h}} D_{\mathrm{m}}-l_{\mathrm{h}}\left(D_{1}+D_{2}\right)=0 .
$$

$\hat{w}(z)$ is given by

$$
\hat{w}(z)= \begin{cases}A_{\mathrm{h}} z, & -D_{1}<z<0, \\ B_{1 \mathrm{~h}} \cos l_{\mathrm{h}} z+B_{2 \mathrm{~h}} \sin l_{\mathrm{h}} z, & -\left(D_{1}+D_{\mathrm{m}}\right)<z<-D_{1}, \\ C_{\mathrm{h}}(D+z), & -D<z<-\left(D_{1}+D_{\mathrm{m}}\right),\end{cases}
$$


where

$$
\frac{B_{2 \mathrm{~h}}}{B_{1 \mathrm{~h}}}=\frac{1+l_{\mathrm{h}} D_{1} \tan l_{\mathrm{h}} D_{1}}{\tan l_{\mathrm{h}} D_{1}-l_{\mathrm{h}} D_{1}}
$$

and $A_{\mathrm{h}}$ and $C_{\mathrm{h}}$ are given by continuity of $\hat{w}(z)$ at the junctions between the layers. The Rossby deformation radius $a$ is given by $a=\left(1-\omega^{2} / f^{2}\right)^{1} /{ }^{2} / K$, and the gravity and Kelvin wave speed is $C_{\mathrm{K}}=a f$. The baroclinic pressure field $p_{i}$ and radial velocity component $u_{r i}$ are given by

$$
p_{i}=\mathrm{i} \rho \frac{f^{2}-\omega^{2}}{\omega K^{2}} \frac{\partial w}{\partial z}, \quad u_{r i}=\frac{\mathrm{i}}{\rho\left(f^{2}-\omega^{2}\right)}\left(\omega \frac{\partial p_{i}}{\partial r}-\frac{f m}{r} p_{i}\right) .
$$

To complete the solution we need to invoke two boundary conditions at $r=r_{\mathrm{a}}$, namely the continuity of the pressure in the upper layer, which gives

$$
\frac{p_{i}\left(r_{\mathrm{a}}\right)}{\rho}+g \eta\left(r_{\mathrm{a}}-\right)=g \eta\left(r_{\mathrm{a}}+\right)
$$

and the blocking of the radial flow in the lowest layer, which gives

$$
\frac{\mathrm{i} A_{1} m}{D} r_{\mathrm{a}}^{m-1}+\frac{1}{K}\left(I_{m}^{\prime}\left(K r_{\mathrm{a}}\right)-\frac{f m}{\omega K r_{\mathrm{a}}} I_{m}\left(K r_{\mathrm{a}}\right)\right) C_{\mathrm{h}}=0 .
$$

Together these give the eigenvalue equation for $m$ :

$$
\frac{m f}{\omega-m \Omega_{0}}\left(1+\frac{2 \Omega_{0}}{f}-\frac{D_{\mathrm{s}}}{D}\left(1+\frac{\omega}{f}\right)+\frac{D_{\mathrm{s}}}{D} \frac{\left(1-\omega^{2} / f^{2}\right)}{T_{1} T_{2}}\right)+\Im(m)=0,
$$

where

$$
\begin{aligned}
T_{1}= & \frac{\omega}{f}\left(\frac{K r_{\mathrm{a}} I_{m-1}\left(K r_{\mathrm{a}}\right)}{m I_{m}\left(K r_{\mathrm{a}}\right)}-1\right)-1, \\
T_{2}= & \frac{D D_{1}}{D_{\mathrm{s}} D_{2}} \cos \left(l_{\mathrm{h}} D_{1}\right) \cos \left(l_{\mathrm{h}}\left(D_{1}+D_{\mathrm{m}}\right)\right) \\
& \times\left[1-\frac{\tan \left(l_{\mathrm{h}} D_{1}\right)}{l_{\mathrm{h}} D_{1}}+\frac{\left(\tan \left(l_{\mathrm{h}} D_{1}\right)+\left(1 / l_{\mathrm{h}} D_{1}\right)\right)\left(l_{\mathrm{h}} D_{1}+\tan \left(l_{\mathrm{h}} D_{\mathrm{m}}\right)\right)}{1-l_{\mathrm{h}} D_{1} \tan \left(l_{\mathrm{h}} D_{\mathrm{m}}\right)}\right]
\end{aligned}
$$

and

$$
\mathfrak{I}(m)=\frac{r_{\mathrm{a}}\left(\mathrm{d} \Psi_{\mathrm{s}}\left(r_{\mathrm{a}}\right) / \mathrm{d} r\right)}{\Psi_{\mathrm{s}}\left(r_{\mathrm{a}}\right)}
$$

If $S \neq 0$, the solutions for $m$ are complex, and we write $m=m_{r}+\mathrm{i} m_{i}$, where the real part $m_{r}$ relates to the wave speed, and the imaginary part $m_{i}$ to the viscous decay. There is some arbitrariness in the choice of these junction conditions. Here, the flow of fluid onto the shelf may be from both the upper and middle layers. In contrast, in the version described in BBXM, the onshelf flow was specified as being entirely in the upper layer. The present version is preferred here, as being a more realistic representation of the experimental conditions. The flow over the shelf, however, is assumed to be barotropic. 
In order to determine the wavespeeds and structure of CTW modes, one first determines $l_{\mathrm{h}}, K, T_{1}$ and $T_{2}$ from (2.22), (2.23), (2.30) and (2.31), and then obtains $m$ for given $\omega$, $\Omega_{0}$. The values for the angular speed are then given by $\omega / m$ (or $\left.\omega / m_{r}\right)$, and the speeds at radius $r$ are given by $\omega r / m$. Although the expressions are different, at the radii of these experiments the values of the CTW speeds at the shelf break, $\omega r_{\mathrm{a}} / m$, are generally similar to the rectilinear speeds given by Gill and Clarke (1974), which they approach in the limit of infinite radius and two-layer stratification at the shelf break.

This solution may be regarded as consisting of an interfacial Kelvin wave in the deep water, coupled to a barotropic Rossby wave on the shelf. There is an infinite number of possible horizontal modes on the shelf, and an infinite number of possible vertical modes in the deep water, but only the first mode in each case is relevant to the experiments. Two parameters that are helpful in characterizing the waves are the Kelvin wave speed $C_{\mathrm{K}}$, and the speed of "pure" shelf waves, $C_{\mathrm{S}}$ at long wavelength. The latter is determined by applying an open boundary condition $\eta=0$ (Clarke and Brink, 1985) to the inviscid shelf wave (2.18) at $r=r_{\mathrm{a}}$, which gives

$$
C_{\mathrm{S}}=\frac{4 f r_{\mathrm{a}}}{\mu\left(1+X^{2}\right)}
$$

where $X$ is a dummy variable defined by $X^{2}+2 X \cot \left(X \mu \ln \left(r_{\mathrm{a}} / r_{\mathrm{b}}\right) / 2\right)=1$. The relative magnitudes of $C_{\mathrm{K}}$ and $C_{\mathrm{S}}$ indicate which is the more important process. The form of the dependence of wave speeds on paddle frequency for inviscid experimental conditions always has the same monotonic form shown in Fig. 1a. Speeds from the viscous theory are all slightly larger than the inviscid speeds (i.e. $m_{r}$ is slightly less than the inviscid value of $m$ ). If a zonal current is present on the shelf, this current reduces the wave speed if it opposes propagation, and increases it if the directions are the same. This effect of the zonal current on wavespeed is less than would be obtained by the advective effect alone, because the advection only applies over the shelf, and not in deep water. It is also weaker when it opposes propagation than when it supports it. An example is shown in Fig. 1b. The model is only valid for small advection velocities within a range such as that shown in Fig. 1 b.

\section{Experiments}

The experiments were conducted in a rotating cylindrical Plexiglas tank of diameter $1.82 \mathrm{~m}$, and total height $20 \mathrm{~cm}$, with the continental shelf/slope topography placed around the periphery. This topography had the form described in the previous section, with the coastline at radius $r_{\mathrm{b}}=91 \mathrm{~cm}$. Two forms were used. The first, narrow form had a continental shelf of exponential profile given by (2.1) of width $10 \mathrm{~cm}$ with parameter $\lambda=0.1$, and a vertical continental slope of height $10 \mathrm{~cm}$ at $r_{\mathrm{a}}=81 \mathrm{~cm}$. The second form had a wider shelf with profile also given by (2.1) but with $\lambda=0.05$, and a vertical continental slope at $r_{\mathrm{a}}=71 \mathrm{~cm}$. No topography was present for $0<r<r_{\mathrm{a}}$ for either form. For the wide shelf runs, the total depth was $15 \mathrm{~cm}$, and for the narrow shelf, runs were conducted with $D=15$ and $17 \mathrm{~cm}$. The tank was rotated in an anti-clockwise direction (northern hemisphere rotation) with a period of $20 \mathrm{~s}$ for all the experiments described here. This rotation gave a variation in the 

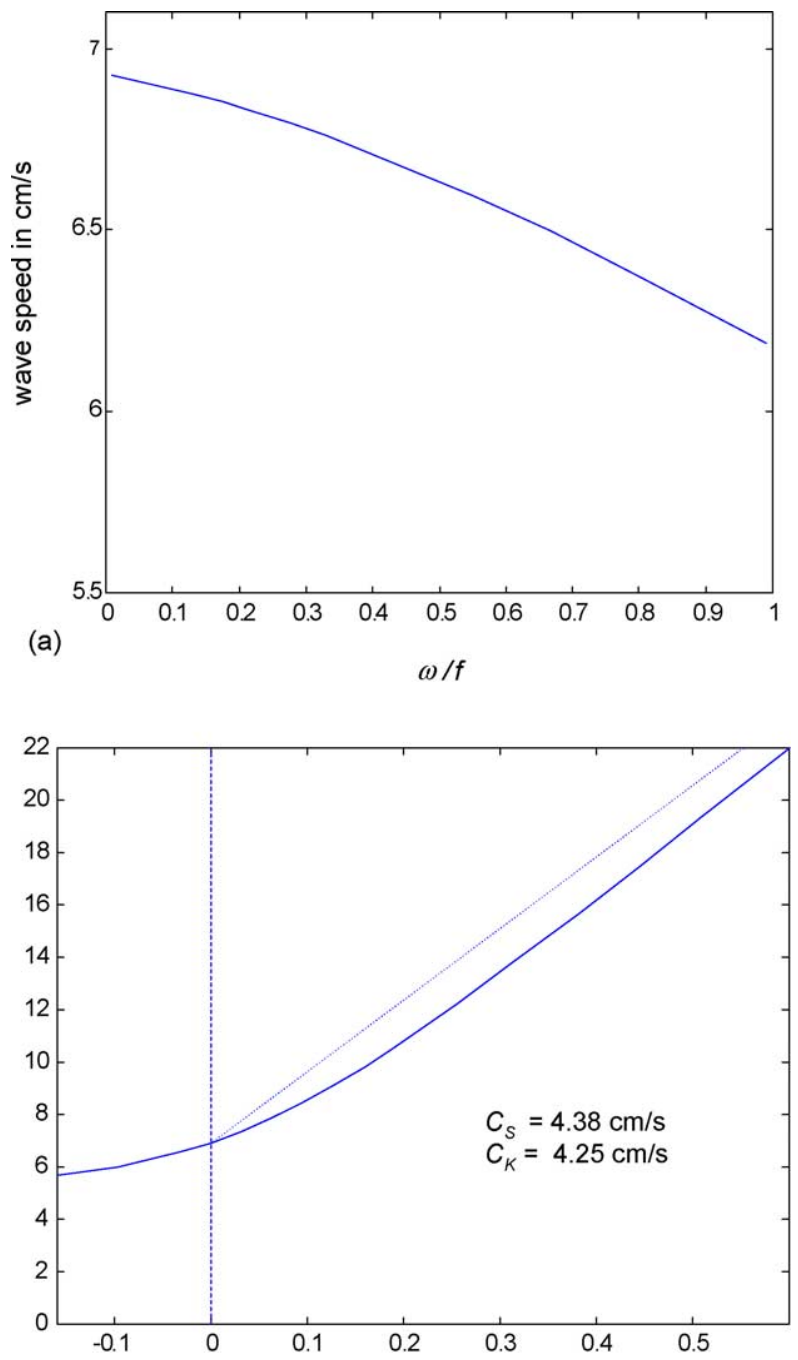

(b)

Fig. 1. (a) An example of typical wave speed dependence on paddle frequency, as calculated from inviscid theory. Narrow shelf, $C_{\mathrm{K}}=4.25 \mathrm{~cm} \mathrm{~s}^{-1}, C_{\mathrm{S}}=4.38 \mathrm{~cm} \mathrm{~s}^{-1}$. (b) An example of the effect of a current on the shelf on wave propagation, from inviscid theory. The dashed line shows the effect of pure advection by a uniform current of the same speed as that represented by the abscissa.

free surface of $4 \mathrm{~mm}$ overall, but less than $1 \mathrm{~mm}$ over the width of the continental shelf. Accordingly, the mean surface height over the shelf was taken as determining the fluid depth, and the paraboloidal free surface variations were otherwise ignored.

The tank was filled to an overall depth $D$ of 15 or $17 \mathrm{~cm}$ using salt-stratified water, to obtain a density-stratified profile. This was done slowly, and took several hours. The initial objective during filling was to obtain as near to a two homogeneous layer density structure 
as possible, with a thin stratified layer in between. Because of the difficulties of a stratified fill in a rotating tank, this was generally imperfectly realized, and the result was typically a three-layer fill with a uniformly stratified region between the homogeneous top and bottom layers. This stratified layer was at a level that was approximately centered at the level of the shelf break, $10 \mathrm{~cm}$ above the floor of the tank, and had a typical overall thickness of $3-4 \mathrm{~cm}$. Experiments with wave generation were not commenced until we were satisfied that the flow was as close to rigid rotation as could be obtained under the circumstances. One problem was that the drag of the air in the laboratory tended to cause observable motion on the surface. To remove this, the superstructure and fluid in the tank were covered by plastic sheeting during spin-up and the experiments. However, some weak residual flow was almost always present.

A number of different ways of generating coastally trapped waves in this geometry were tried for these experiments, and the methods finally adopted were the following. The waves were forced by a radially moving paddle that spanned the whole depth, oscillating with periodic sinusoidal motion near the shelf break, forcing fluid onto the shelf. This paddle had length $97.2 \mathrm{~cm}$, had curvature less than the tank at radius $r_{\mathrm{a}}$, and was flexible. The ends of this paddle were in contact with the continental slope throughout each cycle, though the central part moved radially toward and away from it. This design had the advantage of minimising eddy generation and mixing at the ends of the paddle, whilst still forcing fluid onto and off the shelf, which was the main action that generated CTWs. In contrast, on the deep-water side this paddle motion had no conspicuous effect because the forced motion there was barotropic. Waves were generated with a range of frequencies less than $f$; specifically, paddle periods for individual runs ranged from 80 down to $11 \mathrm{~s}$, slightly above the inertial period of $10 \mathrm{~s}$. Peak to peak paddle amplitudes ranged from 2 to $5 \mathrm{~cm}$. This geometry did not generate ideal sinusoidal CTWs, although these were approximated when the paddle length was approximately one-half the wavelength of the generated wave. The density difference between the upper and lower layers was not varied in a systematic manner, and was constrained to values where $\Delta \rho / \rho_{1}$ was of order 0.003 , in order to maintain a realistic coupled coastal-trapped wave structure.

The waves were observed by using three conductivity probes situated at the shelf break. The first (probe 1) was (typically) at a distance of $86.5 \mathrm{~cm}$ from the center of the paddle, the second (probe 2) a further $81.3 \mathrm{~cm}$, and the third (probe 3) a further $127.3 \mathrm{~cm}$, measured anti-clockwise around the shelf break radius $(80 \mathrm{~cm})$. A diagram of this experimental arrangement is shown in Fig. 2. For some runs the third probe did not function correctly, so that there were effectively two probes. In others there were only two probes. These probes gave measurements of the vertical displacements of the density surfaces, and of the shape of the wave profiles, since their location in the vertical was approximately near the center of the stratified middle layer. Hence they could be used to measure wave speeds and associated wave lengths, amplitudes, and decay rates. In some runs the continental slope topography between the last (third) probe and the paddle was removed, in order to prevent CTWs from propagating to the paddle from "behind", and complicating the interpretation. However, this appeared to make little difference as the amplitudes of the CTWs at probe 3 were always small.

The waves were also observed by placing beads on the free surface, and tracking these by video camera at a location between probes 1 and 2, with the center of the field of view 


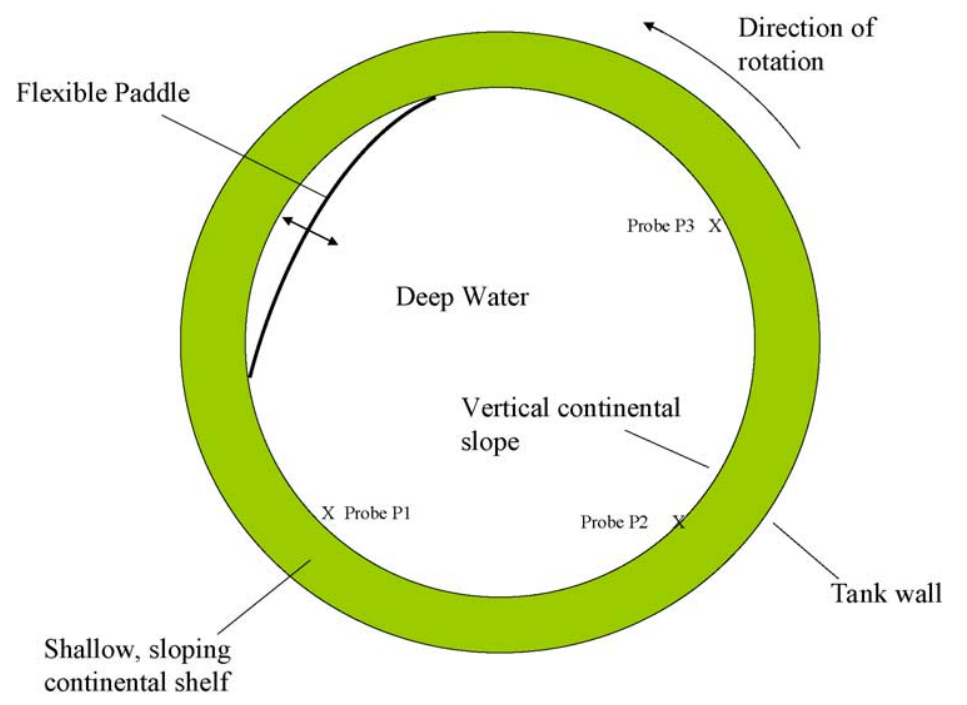

Fig. 2. Schematic plan of the tank and a typical experimental configuration.

approximately $25 \mathrm{~cm}$ upstream of probe 2 . The tank was covered with a superstructure that was wrapped in plastic sheeting to minimise any effect of drag of the air on the free surface, and from air currents in the laboratory. This could not be totally successful, but some comparisons with beads and tracers below the surface indicated that, for the most part, the beads floating on the surface gave a reasonably faithful representation of the motion of the top layer as a whole. This enabled the observation of flow fields in the upper layer. These were seen to be qualitatively consistent with theory, but quantitative comparisons were not made.

Over 100 runs were carried out that produced useful data, in approximately 13 series, in each of which the paddle frequency was varied from a low value $(80 \mathrm{~s}$ period) to the maximum. These runs covered variations in the forcing paddle frequency, paddle amplitude, stratification, total depth, and shelf width. Paddle length and the rotation rate of the tank were kept constant for all these experiments.

\section{Results}

We begin with a description of the observed flow that is representative of each series of runs undertaken. The vertical displacements measured by the conductivity probes at the shelf-break tended to show repeatable oscillating patterns at each probe for each run, but with considerable variations between the runs. We have taken this variable as a measure of the amplitude of the disturbances produced, given that it is visually correlated with the flow pattern on the shelf as described above. The nature of the conductivity record depended on the stratification as well as the other parameters, and this produced a lot of variety in the records. Wave amplitudes varied during the records, and in some 

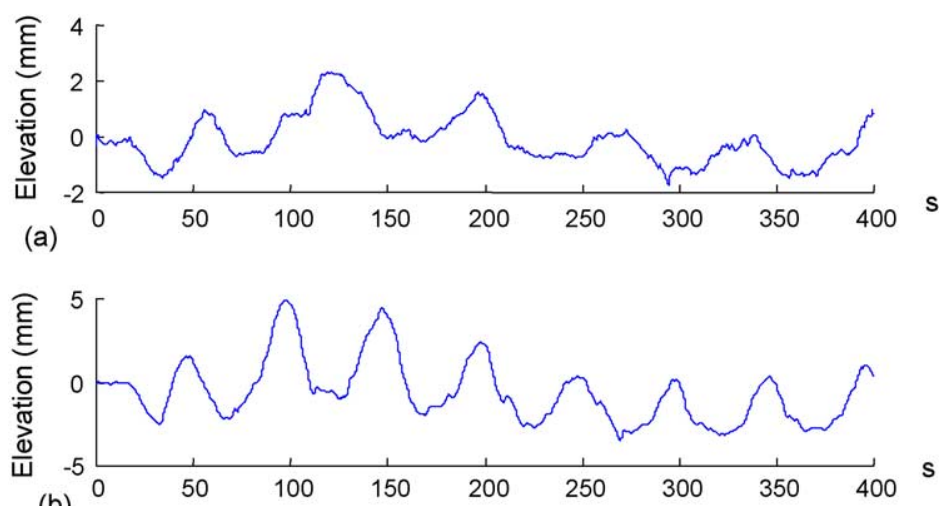

(b)

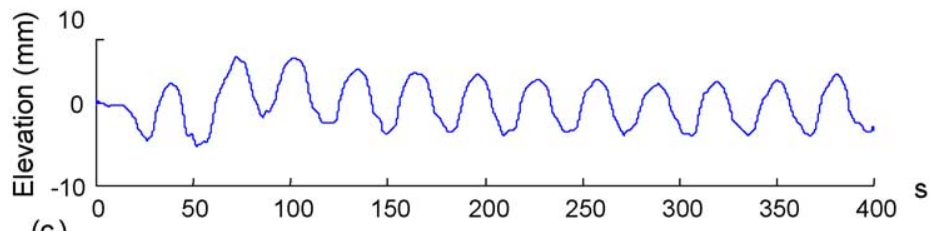

(c)

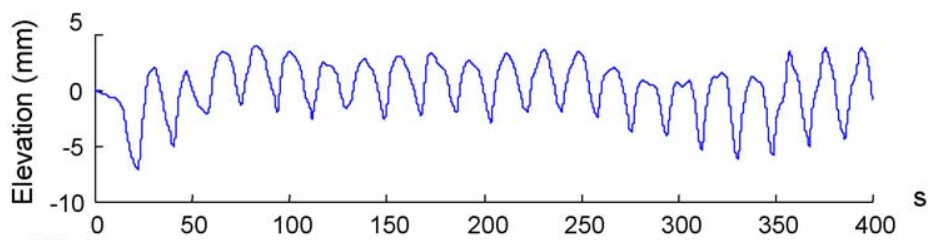

(d)

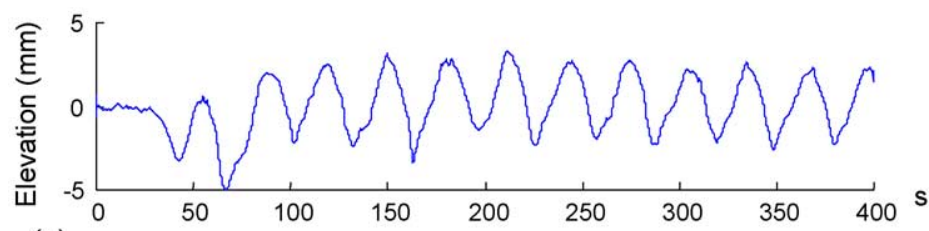

(e)

Fig. 3. Typical wave profiles, as measured by the conductivity probes. Wide shelf $(20 \mathrm{~cm})$, paddle amplitude $5 \mathrm{~cm}$ (peak to peak), $D=15 \mathrm{~cm}, C_{\mathrm{S}}=8.17 \mathrm{~cm} \mathrm{~s}^{-1}, C_{\mathrm{K}}=4.25 \mathrm{~cm} \mathrm{~s}^{-1}$. Along-shelf distances-center of paddle to probe $1: 157 \mathrm{~cm}$, probe 1 to probe $2: 110 \mathrm{~cm}$. (a) $\omega / f=0.145$ (paddle period $69 \mathrm{~s}$ ), from probe 1 ; (b) $\omega / f=0.204$, probe1; (c) $\omega / f=0.323$, probe 1 ; (d) $\omega / f=0.556$, probe 1 ; (e) $\omega / f=0.323$, probe 2 . Note the changes in scale.

cases were initially large and then almost disappeared. However, the following description applies to most of the series of runs observed. Some typical traces are shown in Fig. 3, which are all for the same stratification. Here the variable plotted is vertical displacement, which has been inferred from the fluid density variation provided that the amplitude is not too large, and the probe remains within the stratified "mixed" region of the fluid. Sufficiently large displacements can result in flat-topped density profiles if the region of density variation moves above or below the probe. This has rarely been a problem in these 
experiments, and the waves are not large enough to affect the profiles in Fig. 3 in this manner.

At small values of $\omega / f$, as in Fig. 3a, the wave amplitude is small and the shape is not well formed. This is because the length of the forcing paddle $L_{\mathrm{P}}(97.2 \mathrm{~cm})$ is much less than half the total wavelength $L_{\mathrm{W}}(470 \mathrm{~cm}$ in the case of Fig. 3a), and this mismatch is not efficient for wave generation. If the experiments are repeated with increased frequency so that $L_{\mathrm{W}}$ shortens, the wave amplitude increases and the waves become progressively more sinusoidal as shown in Fig. $3 \mathrm{~b}$ and c, until $L_{\mathrm{W}}<2 L_{\mathrm{P}}$. Wave amplitudes then decrease again as $\omega / f$ approaches unity, and the profiles become (vertically) asymmetric, with steep troughs and broad crests, as shown in Fig. 3d. In Fig. 3c, the wave is the closest to sinusoidal, but this profile evolves with further propagation to have steep troughs and relatively broader crests as seen in Fig. 3e. This is the expected form of finite-amplitude, nonlinear internal waves with a shallow upper layer. In general, the wave profiles at higher frequencies (where $\left.L_{\mathrm{W}}<2 L_{\mathrm{P}}\right)$ tend to have broader crests and narrower troughs. All of these curves are interpreted as representing the lowest vertical mode for the internal Kelvin-like waves, and there was no indication in any of these series of runs that higher order vertical modes were present.

Observations of the videos of the upper layer motion confirmed that the pattern of flow over the shelf was consistent with that expected from the theory, and was linked with the vertical displacements at the shelf break. Fluid particles over the shelf moved in an elliptical path in a clockwise direction. Offshore motion (negative $u_{r}$, with fluid motion directed radially inwards) in this elliptical movement coincided with downward motion of the "interface" at the shelf break. At along-slope distances downstream from the paddle greater than about $30 \mathrm{~cm}$, the flow pattern on the shelf was recognizably consistent with that of the lowest theoretical spanwise mode (of which the inviscid form is given by (2.18)), though subject to distortion by nonlinear advection and viscous effects. No significant generation of higher order modes by the paddle was apparent. At low frequencies, the particle displacements on the shelf were approximately periodic, but not generally sinusoidal. As the paddle frequency was increased, the motions became more sinusoidal in time, but also a net prograde drift developed. When the frequency was increased beyond the "resonance frequency" (where the wavelength is twice the paddle length), the temporal motion became increasingly different from sinusoidal. Near the paddle (probe 1), the alongshore motion on the shelf occurred in strong surges of prograde flow, which coincided with the steep downward displacements of the density field shown in Fig. $3 \mathrm{~d}$ (and to a lesser extent, Fig. 3e). The asymmetrical profile typified by Fig. 3d therefore represents the response of the fluid to the forcing by the paddle, which forces these surges at frequencies above resonance. Hence there are two processes that tend to produce waves with steep troughs and broad crests: prograde surges of current on the shelf, and nonlinear gravity wave steepening, though the latter effect is not strong since the displacements are not large.

The wave speeds, as measured by the probes, have been compared with the inviscid theoretical values for all measured runs, and these ratios are shown in Fig. 4. These speeds were measured from plots of the observed traces. The general consistency between these ratios is consistent with these motions being interpreted as periodic CTWs. In some cases there is variability in the speed during the run. There is also considerable variability between 

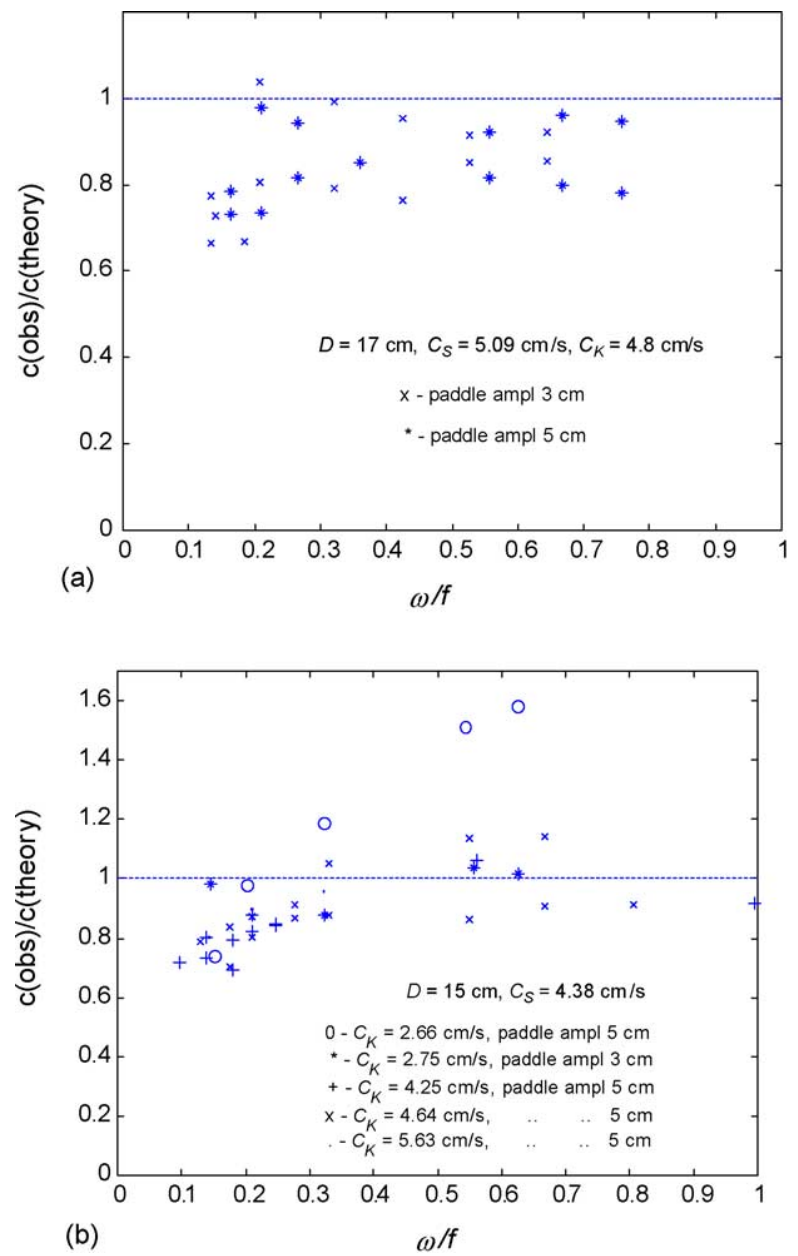

Fig. 4. Wave speeds for the runs studied. (a) Narrow shelf, width $r_{\mathrm{b}}-r_{\mathrm{a}}=10 \mathrm{~cm}$, total depth $D=17 \mathrm{~cm}$. (b) Narrow shelf, width $r_{\mathrm{b}}-r_{\mathrm{a}}=10 \mathrm{~cm}$, total depth $D=15 \mathrm{~cm}$. (c) Wide shelf, width $r_{\mathrm{b}}-r_{\mathrm{a}}=20 \mathrm{~cm}$, total depth $D=15 \mathrm{~cm}$.

the ratios of wave speeds obtained from the different sets of runs. Although the values from some series lie close to unity, in others the value is as low as 0.6. This scatter is most prominent in the runs with the broad shelf (width $20 \mathrm{~cm}$ ). There is some subjectivity in the measurement of these speeds, which is reflected in the scatter. In some cases, the speed varies during the course of a run, and a visual best fit was chosen. However, the main conclusion is that the observed speeds were generally less than expected from the theory. The most likely reason for this appears to be that, in the experiments, the fluid over the shelf was partly stratified, particularly at levels just above the shelf break, whereas in the theory, the fluid over the shelf was assumed to be homogeneous. The presence of stratification over the shelf would have the effect of reducing the vortex stretching, which implies reducing the 
topographic Rossby wave restoring force, and hence in turn the wave speed. We note that this explanation is supported by the observation that this theoretical-experimental discrepancy generally increases with the ratio $C_{\mathrm{S}} / C_{\mathrm{K}}$, and is the largest for the wide shelf runs where the theoretical vortex stretching effect is the largest. It should be noticed that an increase in the overall stratification causes an increase in the wave speeds (Huthnance, 1978), but this is quite consistent with the argument given above for a decrease, since this only involves a re-distribution (spreading) of the isopycnals.

There is evidence that the amplitude of the paddle motion may have some effect on the wave speed when $\omega / f$ is not small. At large amplitude $(\sim 5 \mathrm{~cm})$ with the narrow shelf, the paddle produced a net rectified flow on the shelf in the direction of propagation, which on theoretical grounds (Section 3 and Fig. 1b) is expected to give a net increase in observed wave speed. This is a rectification of the prograde surges associated with the troughs in vertical displacement at the shelf break, described above. This rectified current emanating from the vicinity of the paddle is generally the strongest over the region of shallower depths close to the wall, and does not cause significant shear near the shelf break. In consequence, there was no evidence of shear instability associated with this mean shelf current. There is a general tendency for the observed wave speeds to increase relative to the theoretical ones as $\omega / f$ increases, as shown in Fig. 4. This is most pronounced in the set of runs where $C_{\mathrm{K}}$ is small.

Measurements were made of the offshore structure of the waves in the deep water. From inviscid theory, the vertical velocity $w$ is given by Eq. (2.21), and the vertical displacement $\eta$ by $\eta=-\mathrm{i} \omega w$. Hence the displacement amplitude of the wave is expected to decrease approximately exponentially with distance toward the center of the tank, as described by the Bessel function $I_{m}(K r)$ in (2.21). Fig. 5(a) shows the typical records from three probes on a horizontal radial line spaced $10 \mathrm{~cm}$ apart, with the first at the shelf break, and the others progressively closer to the central axis of the tank. The rapid decrease in amplitude of the signal with distance "seaward" is evident. Fig. 5(b) shows the comparison between the observed amplitudes at these three probes with linear theory. There is good agreement at low frequencies, but at higher frequencies the waves are more tightly confined to the coastal environment than linear theory predicts. It is possible that this nonlinear property is related to the nonlinear profiles of the Kelvin wave-like component of the CTW at higher frequencies, as shown in Fig. 3d and e.

Measurements of the amplitudes of the conductivity variations at the three probes also permitted the computation of the observed decay rates with along-slope distance, and comparison with computed decay from viscous theory of Section 3. The theory gives $m_{i}$ values for a decay factor $\mathrm{e}^{-m_{i} \theta}$, where $\theta$ is the angular distance of propagation. This may be converted into linear distance decay factor $\mathrm{e}^{-k_{\mathrm{d}} x}$, where $x$ is the distance along the shelf break and $k_{\mathrm{d}}=m_{i} / r_{\mathrm{a}}$. Results are expressed in terms of $k_{\mathrm{d}}$, in units of $\mathrm{cm}^{-1}$. The expansions used in the viscous theory do not converge for $S>1$, which occurs when $\omega / f$ is small. However, the computed values of $m_{i}$ vary only slightly with $\omega / f$, and a mean value was adopted for each set of runs with the same stratification. For the wide shelf runs, if we exclude the region $\omega / f<0.2$ where the wave motion is irregular, the theoretical and experimental values are generally consistent. An example of this comparison is given in Fig. 6. Here the motion on the shelf is an important part of the propagation, and the bottom friction effects are significant. For the narrow shelf, on the other hand, the observed decay rates 

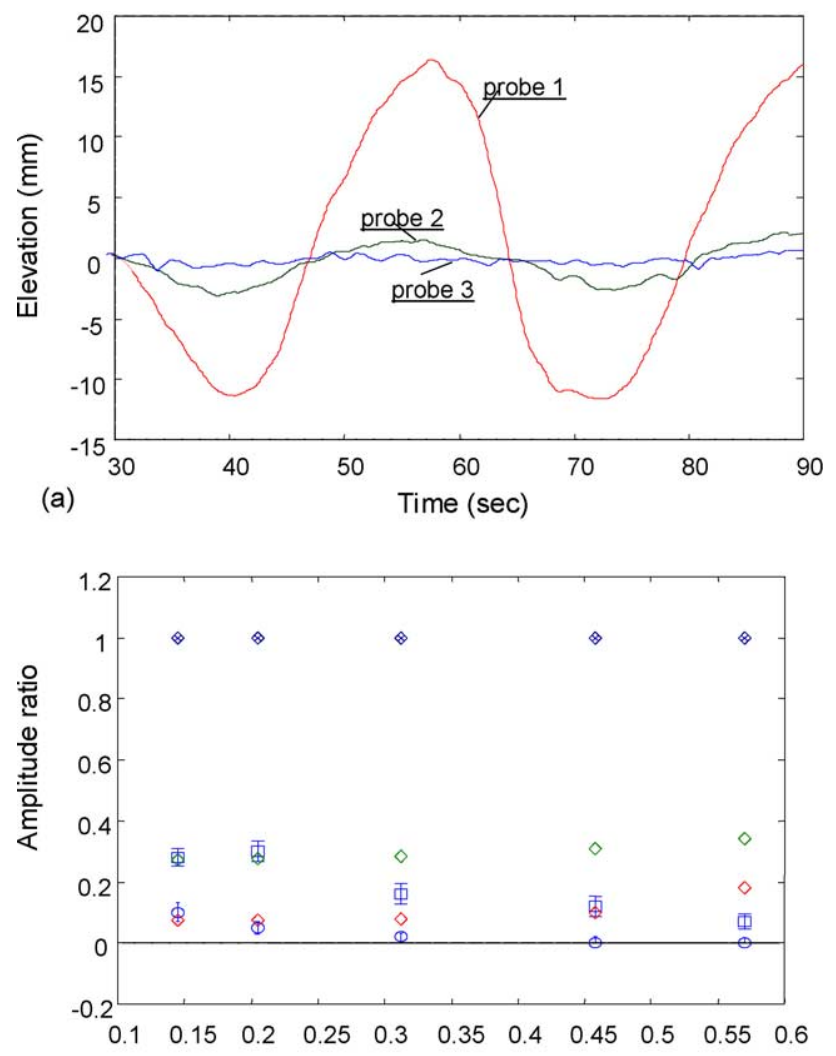

(b)

omega/f

Fig. 5. (a) Time series of isopycnal displacements at three probes in a radial line in the deep water at shelf break depth, for the narrow shelf of width $10 \mathrm{~cm}$. Probe 1 is at the shelf break, probe 2 is $10 \mathrm{~cm}$ closer to the center of the tank, and probe 3,10 cm even closer (i.e. radii of 81,71 and $61 \mathrm{~cm}$, respectively). Paddle period $32.6 \mathrm{~s}, \omega / f=0.307$. (b) Comparison between the amplitudes of the signal at the three probes with linear theory, scaled as unity at the shelf break, as a function of $\omega / f$. The diamond symbols show the theoretical values from (2.21), the squares the amplitude ratios measured at probe 2 , and the circles the amplitude ratios at probe 3 . Error bars show standard deviation over a number of observed periods.

are highly variable and generally much larger than the theoretical values based on surface friction on the shelf, with e-folding decay distances of the order of $1 \mathrm{~m}$ for many runs. At low frequencies, where the theoretical wavelengths are long and the amplitudes are not large anyway, this may be attributed to dispersion of the waves, which are irregular in shape (see Fig. 3a), and the selective removal of small-scale features. Generated waves that do not have the theoretical wavelength will not propagate at the theoretical speed. At high frequencies, the amplitude is largely dependent on the steep troughs associated with the prograde surges, which appear to lose amplitude rapidly with propagation. In general, the wide shelf configuration gave better simulations of idealized CTWs than the narrow shelf. 


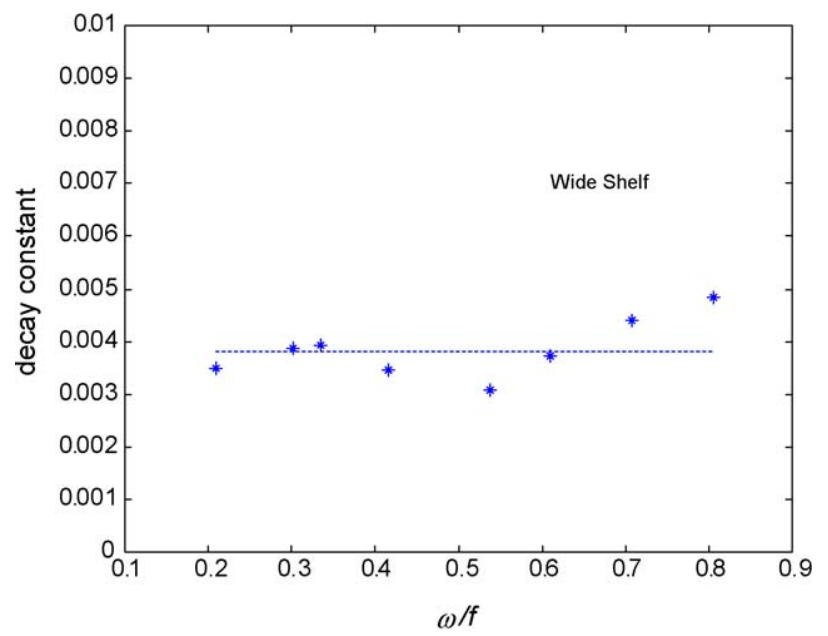

Fig. 6. Observed decay rates $k_{\mathrm{d}}$ (in units of $\left.\mathrm{cm}^{-1}\right)$ for distance along the shelf, for a series of wide shelf $(20 \mathrm{~cm})$ experiments, compared with the theoretical value (dashed line) from the model of Section $3 . C_{\mathrm{S}}=8.17 \mathrm{~cm} \mathrm{~s}^{-1}$, $C_{\mathrm{K}}=3.8 \mathrm{~cm} \mathrm{~s}^{-1}, 4.05 \mathrm{~cm} \mathrm{~s}^{-1}$.

\section{Conclusions}

We have carried out an experimental study of coastal-trapped waves with idealized topography, and compared the results with theoretical expectations. The topography used represents oceanic conditions where the Burger number is large, because the continental shelf and slope are relatively steep. The theoretical model of Gill and Clarke (1974) has been extended to include waves that are not assumed to be long, curved geometry, three-layer stratification with a stratified central layer, an alongshore shelf current, and bottom friction on the shelf. It does, however, assume barotropic motion on the shelf.

The experiments were designed to conform to this theoretical model. The topography consisted of a sloping continental shelf, and a vertical continental slope. The stratification consisted of an approximately homogeneous thick lower and thin upper layers, separated by a stratified middle layer centered near the level of the shelf break. Using a flexible paddle that was placed against the vertical continental slope and oscillated radially, a periodic motion was produced. This arrangement minimized eddy generation at the ends of the paddle, and produced disturbances that had many of the features of coastal-trapped waves in this coastal waveguide, for $0<\omega / f<1$. Videos of the surface flow showed that the particles over the shelf moved in elliptical paths that conformed to the vertical displacements at the shelf break. At the lower end of this range, where $L_{\mathrm{P}} \ll c /(2 \omega)$ (where $c$ is the linear wave speed at the shelf break), the waves were not well formed and the amplitude was small. The amplitude increased with increasing $\omega / f$, until this inequality was reversed and $\omega / f$ approached unity. In the central range of $\omega / f$, the waves were approximately sinusoidal, but as $\omega / f$ approached unity the vertical displacements at the shelf break (at the probe closest to the paddle) had short steep troughs and flat crests, the troughs coinciding with prograde surges in current over the shelf. 
The waves were mostly observed to travel at slower speeds than expected from theory. This effect was larger for wider slopes, and is attributed, at least partly, to the fact that the theory assumes that motion on the shelf is barotropic, whereas it is affected by stratification in the experiments in the range of depths just above the shelf break. The waves were also observed to be damped more rapidly than expected for the narrow shelf. This appears to be because, at the low and high frequency limits the waves are not sinusoidal, and are subject to dispersion at the low end, and nonlinear effects at the high end.

An additional factor that could contribute to the observational wave speeds being less than those predicted by the theory is the use of a laminar drag coefficient for the bottom boundary layer in the theoretical analysis. Lilly (1966) found that a homogeneous geostrophic motion along a horizontal smooth boundary became unstable at a Reynolds number, $\operatorname{Re}_{\delta}=\left(2 u_{\mathrm{G}}^{2} / v f\right)^{1 / 2}>55$, where $u_{\mathrm{G}}$ is the geostrophic velocity outside the Ekman boundary layer on the model floor. Caldwell and Van Atta (1970) found that laboratory experiments were in good agreement with the Lilly (1966) findings in that the flow became unstable at $R e_{\delta}>56.7$, but that the instabilities were in the form of longitudinal rolls; they further found that fully developed turbulence did not occur until $\operatorname{Re}_{\delta}>148$. For turbulent boundary layers the drag coefficient is proportional to $u_{\mathrm{G}}^{2}$, rather than $u_{\mathrm{G}}$ as in the laminar case for transitional boundary layers the drag coefficient will have a more complex representation than either the laminar or the turbulent cases; see Tennekes and Lumley (1972). For the present experiments $v=0.01 \mathrm{~cm}^{2} \mathrm{~s}^{-1}$ and $f=0.63 \mathrm{~s}^{-1}$. Transitional flow is thus expected for velocities $u, v>3.1 \mathrm{~cm}^{-1}$, but because of the complex topography transition could occur at substantially smaller flow speeds. Such large velocities are generated by the paddle at the higher ends of the range of amplitudes and frequencies used in the experiments. Hence the drag may be larger than predicted and, in consonance with the theory, lead to correspondingly lower wave speeds.

In general, these experiments have successfully produced coastal-trapped waves (CTWs) in the laboratory, over a wide range of conditions. We have identified various techniques that are useful for generating and modeling them, and explored the limits of these techniques and the waves so produced. They provide a basis for an extension of these studies into the hydraulic regime where advective effects on the waves may be explored.

Furthermore, the comparisons between the laboratory data and the theoretical model again show the importance of using the laboratory as a benchmark for analytical and/or numerical models, or for that matter, field experiments, each of which, as do the laboratory experiments, has their shortcomings in the overall effort to develop a better understanding of ocean processes.

\section{References}

Abramowitz, M., Stegun, I.A., 1965. Handbook of Mathematical Functions, Dover, 1046 pp.

Allen, J.S., 1980. Models of wind-driven currents on the continental shelf. Annu. Rev. Fluid Mech. 12, $389-433$.

Baines, P.G., Boyer, D.L., Xie, B., Murray, D.L., 2001. Laboratory observations of coastally trapped waves. In: Proceedings of the Third International Symposium on Environmental Hydraulics, Arizona State University, Tempe, AZ, USA, p. 6.

Battisti, D.S., Hickey, B.M., 1984. Application of remote wind-forced coastal trapped wave theory to the Oregon and Washington coasts. J. Phys. Oceanogr. 14, 887-903. 
Brink, K.H., 1982. The effect of bottom friction on low-frequency coastal-trapped waves. J. Phys. Oceanogr. 12, $127-133$.

Brink, K.H., 1990. On the damping of free coastal-trapped waves. J. Phys. Oceanogr. 20, 1219-1225.

Brink, K.H., 1991. Coastal-trapped waves and wind-driven currents over the continental shelf. Annu. Rev. Fluid Mech. 23, 389-412.

Brink, K.H., 1998a. Wind-driven currents over the continental shelf. In: Brink, K.H., Robinson, A.R. (Eds.), The Sea, vol. 10. Wiley, pp. 3-20, Chapter 1.

Brink, K.H., 1998b. Deep-sea forcing and exchange processes. In: Brink, K.H., Robinson, A.R. (Eds.), The Sea, vol. 10. Wiley, pp. 151-167, Chapter 6.

Brink, K.H., Chapman, D.C., 1987. Programs for computing properties of coastal-trapped waves and wind-driven motions over the continental shelf and slope, 2nd ed. Rep. No. WHOI-87-24. Woods Hole Oceanog. Inst., Woods Hole, MA, $119 \mathrm{pp}$.

Caldwell, D.R., Van Atta, C.W., 1970. Characteristics of Ekman boundary layer instabilities. J. Fluid Mech. 44 (Part 1), 79-95.

Chapman, D.C., 1987. Application of wind-forced, long, coastal trapped wave theory along the California coast. J. Geophys. Res. 92, 1798-1816.

Church, J.A., Freeland, H.J., 1987. The energy source for coastal-trapped waves in the Australian coastal experiment region. J. Phys. Oceanogr. 17, 231-247.

Church, J.A., White, N.J., Clarke, A.J., Freeland, H.J., Smith, R.L., 1986. Coastal-trapped waves on the east Australian Continental shelf. Part II. Model verification. J. Phys. Oceanogr. 16, 1945-1957.

Clarke, A.J., Brink, K.H., 1985. The response of stratified frictional flow of shelf and slope waters to fluctuating large-scale low-frequency wind forcing. J. Phys. Oceanogr. 15, 439-453.

Clarke, A.J., Van Gorder, S., 1986. A method for estimating wind-driven frictional time-dependent, stratified shelf and slope water flow. J. Phys. Oceanogr. 16, 1013-1028.

Clarke, A.J., Ahmed, R., 1999. Dynamics of remotely forced intraseasonal oscillations off the western coast of South America. J. Phys. Oceanogr. 29, 240-258.

Codiga, D.L., Renouard, D.P., Fincham, A.M., 1999. Experiments on waves trapped over the continental slope and shelf in a continuously stratified rotating ocean, and their incidence on a canyon. J. Mar. Res. 57, 585-612.

Crawford, W.R., Thomson, R.E., 1984. Diurnal period continental shelf waves along Vancouver Island: a comparison of observations with theoretical models. J. Phys. Oceanogr. 14, 1629-1646.

Csanady, G., 1978. The arrested topographic wave. J. Phys. Oceanogr. 8, 47-62.

Daifuku, P.R., Beardsley, R.C., 1983. The K1 tide on the continental shelf from Nova Scotia to Cape Hatteras. J. Phys. Oceanogr. 13, 3-17.

Enfield, D.B., Camego-Rodriquez, M., Smith, R.L., Newberger, P.A., 1982. The equatorial source of propagating variability along the Peru coast during the 83 El Nino. J. Geophys. Res. 92 (14), 335-346.

Flather, R.A., 1988. A numerical investigation of tides and diurnal-period continental shelf waves along Vancouver Island. J. Phys. Oceanogr. 18, 115-139.

Freeland, H.J., Boland, F.M., Church, J.A., Clarke, A.J., Forbes, A.M.G., Huyer, A., Smith, R.L., Thompson, R.O.R.Y., White, N.J., 1986. The Australian coastal experiment: a search for coastal-trapped waves. J. Phys. Oceanogr. 16, 1230-1249.

Garrett, C.J.R., MacCready, P., Rhines, P.B., 1993. Boundary mixing and arrested Ekman layers: rotating stratified flow near a sloping boundary. Annu. Rev. Fluid Mech. 25, 291-323.

Gill, A.E., Clarke, A., 1974. Wind-induced upwelling, coastal currents and sea level changes. Deep Sea Res. 21, 325-345.

Hamon, B.V., 1962. The spectrums of mean sea level at Sydney, Coffs Harbour, and Lord Howe Island. J. Geophys. Res. 67 (13), 5147-5155.

Hamon, B.V., 1963. Correction to "The spectrums of mean sea level at Sydney, Coffs Harbour, and Lord Howe Island". J. Geophys. Res. 68 (15), 4635.

Hamon, B.V., 1966. Continental shelf waves and the effects of atmospheric pressure and wind stress at sea level. J. Geophys. Res. 71 (12), 2883-2893.

Huthnance, J.M., 1978. On coastal trapped waves: analysis and numerical calculation by inverse iteration. J. Phys. Oceanogr. 8, 74-92.

Lilly, D.K., 1966. On the instability of the Ekman boundary layer. J. Atmos. Sci. 23, 481-494. 
Mitchum, G.T., Clarke, A.J., 1986. The frictional nearshore response to forcing by synoptic scale winds. J. Phys. Oceanogr. 16, 1029-1037.

Noble, M., Butman, B., 1979. Low frequency wind-induced sea level oscillations along the east coast of North America. J. Geophys. Res. 84, 3227-3236.

Rhines, P.B., 1970. Edge, bottom and Rossby waves. Geophys. Fluid Dyn. 1, 273-302.

Robinson, A.R., 1964. Continental shelf waves and the response of sea level to weather systems. J. Geophys. Res. 69 (2), 367-368.

Smith, R.L., 1978. Poleward propagating perturbations in currents and sea level along the Peru coast. J. Geophys. Res. 83, 6083-6092.

Tennekes, H., Lumley, J.L., 1972. A First Course in Turbulence. MIT Press.

Yankovsky, A.E., Garvine, R.W., 1998. Subinertial dynamics on the inner New Jersey shelf during the upwelling season. J. Phys. Oceanogr. 28 (2), 444-458.

Wang, D.-P., Mooers, C.N.K., 1976. Coastal trapped waves in a continuously stratified ocean. Phys. Oceanogr. 6, 853-863.

Whitehead, J.A., Chapman, D.C., 1986. Laboratory observations of a gravity current on a sloping bottom: the generation of shelf waves. J. Fluid Mech. 172, 373-399.

Wilkin, J.L., Chapman, D.C., 1987. Scattering of continental shelf waves at a discontinuity in shelf width. J. Phys. Oceanogr. 17, 713-724.

Wilkin, J.L., Chapman, D.C., 1990. Scattering of coastal-trapped waves by irregularities in coastline and topography. J. Phys. Oceanogr. 20, 396-421. 